\title{
Rac1 is correlated with aggressiveness and a potential therapeutic target for gastric cancer
}

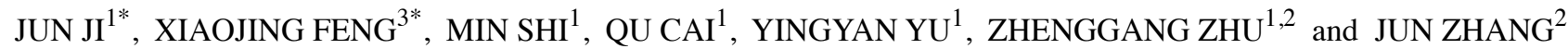 \\ Departments of ${ }^{1}$ Surgery, ${ }^{2}$ Clinical Oncology, and ${ }^{3}$ Clinical Laboratory, Ruijin Hospital, \\ Shanghai Jiaotong University School of Medicine, Shanghai 200025, P.R. China
}

Received November 17, 2014; Accepted January 5, 2015

DOI: 10.3892/ijo.2015.2836

\begin{abstract}
Rac1 is a member of the Rho GTPase family. Racl activity is critical in regulating cytoskeleton organization and thus, modulates a diverse spectrum of cellular functions in normal and malignant cells. The aims of the present study were to investigate the expression pattern and clinical significance of Rac1, as well as the role of Rac1 in gastric cancer tumorigenesis and metastasis. The expression of Rac1 in human gastric cancer was explored by immunohistochemistry. The correlation of Rac1 expression with the clinicopathological characteristics and the survival of patients were analyzed by Pearson's Chi-square and KaplanMeier analyses, respectively. Racl overexpression cell model was used to examine in vitro and in vivo effects of Rac1 in cell growth, migration and invasion. Rac1 was highly expressed in gastric cancer tissues and correlated with differentiation, local invasion, lymph node metastasis and Lauren's classification. Rac1 expression in gastric cancer predicted shorter survival. Overexpression of Rac1 in gastric cancer cells dramatically induced Racl activation and rendered a more aggressive phenotype such as increased cell growth and migration/invasion in vitro and in vivo. Inhibiting Rac1 activity by specific inhibitor abrogated the effects of Rac1 on the malignant phenotype. Our clinical findings demonstrated that Rac1 was well correlated with aggressiveness and a negative prognostic factor. In addition, our data on experimental cell models supported the fundamental role of Rac1 in gastric cancer. Given its pivotal role in gastric tumorigenesis and progression, Racl can serve as a promising therapeutic target for gastric cancer.
\end{abstract}

Correspondence to: Dr Jun Zhang, Department of Clinical Oncology, Ruijin Hospital, Shanghai Jiaotong University School of Medicine, No. 197 Ruijin Er Road, Shanghai 200025, P.R. China E-mail: jun_zj10977@163.com

${ }^{*}$ Contributed equally

Key words: Rac-1, gastric cancer, metastasis, tumorigenesis, prognosis

\section{Introduction}

Gastric cancer is the fourth most common cancer worldwide and is the second most common cause of cancer-related deaths. Due to lack of specific symptoms, gastric cancer patients are often diagnosed at advanced stage. Advanced gastric cancer has a poor prognosis (1) due to its high rate of metastasis. The identification of predictive markers for cancer progression and prognosis would facilitate evaluating the clinical outcome and potential treatment stratification for patients with gastric cancer. More importantly, identification of such markers with biological functions that are critical in gastric tumorigenesis and progression will also provide targets for cancer specific treatment.

Rho GTPases are sensitive molecular switches existing either in an inactive, GDP-bound form or an active GTP-bound form. They are endowed with GTP hydrolytic activity, mainly involved in cytoskeleton rearrangements and cell motility, but also involved in cell proliferation, transformation and differentiation (2). The exchange of GDP to GTP and thus the activation of Rho GTPases are catalyzed by guanine nucleotide exchange factors (GEFs), which act as downstream factors for many extracellular growth factors. Thus, Rho GTPases are key integrating molecules from different extracellular signals. When activated, Rho GTPases can interact and activate a large panel of effectors that are responsible for regulating critical cellular functions. Members of the Rho small GTPases family, prototype RhoA, Racl and Cdc42, are involved in the regulation of a variety of cellular processes, such as organization of the microfilament network, cell-cell contact, and malignant transformation and also perform essential and specialized functions during organization of the actin cytoskeleton.

Ras-related C3 botulinum toxin substrate 1 (Rac1) is a member of the small molecule G-protein Rho family (Ras homologue) and is an important class of intracellular signaling molecules. RAC1 activity, as a modulator of the cytoskeleton, is critical for a number of normal cellular activities including phagocytosis, mesenchymal-like migration, axonal growth, adhesion and differentiation of multiple cell types. Racl also plays key roles in transmitting upstream signaling, such as receptors for extracellular growth factors (3) and receptor tyrosine kinases (RTKs) (4), into downstream cascades and, thus, modulates cellular functions. Upon activation, Rac1 interacts with various specific effectors to coordinate the activation 
of a multitude of signaling cascades that influence diverse physiological outcomes. For example, Pak1 binds to Rac1 in a GTP-dependent manner, after which activated Pak1 regulates cellular functions such as cytoskeletal dynamics, cell adhesion and transcription (5). Rac1 signals can also activate MAPK family member including p38, p42/44 and c-Jun N-terminal kinase (JNK) (6-9). Rac1 was found being able to elevate cytosolic and nuclear levels of $\beta$-catenin, thus, activated Wnt signaling cascades (10).

Although Racl as a cytoskeleton organizer has been documented well, more and more recent findings provoked broad functions of Rac1 in human cancers. Rac1 regulates a diverse spectrum of cellular functions including tumorigenesis $(11)$, angiogenesis $(12,13)$ and metastasis $(11,14,15)$. Overexpression of Rac1 occurs in several different types of tumors including breast $(16,17)$, colon (18), bladder (19), and gastric cancer $(20,21)$. Rac1 overexpression was observed in metastatic gastric (21), breast (15), prostate (22), bladder upper urinary tract cancer (19). Rac1 promoted prostate cancer progression and recurrence upon activation by VAV3, a GEF for Racl activation (14). Rac1 is also involved in sustained cell growth (23) and therapy resistance (24) through crosstalk with other signaling pathways. Racl regulates vasculogenesis and angiogenesis, two distinct but related steps in vasculature. Thus, Racl has been shown closely related with tumor angiogenesis and microvascular density (13). As Rac1 is intimately involved in broad range of cellular functions in human cancers and emerging as a potential target for anticancer therapies, we explored the clinical correlation of Racl in gastric cancer specimens and its biological impacts on gastric cancer malignancy. Our studies suggest that Rac1 is correlated with an aggressive phenotype in gastric cancer and may serve as a target against gastric cancer.

\section{Materials and methods}

Patients and specimens. Gastric cancer tissues, confirmed by pathological diagnosis, were obtained from 92 patients who underwentradical resection for gastric cancer between 2006 and 2008 at the Department of Surgery, Ruijin Hospital, Shanghai, China. The corresponding non-tumor gastric tissue was obtained at least $6 \mathrm{~cm}$ from the tumor. All tissue samples were formalin-fixed and paraffin-embedded. Clinicopathological and survival data of all patients were collected. TNM staging was classified based on the criteria of the American Joint Committee on Cancer (AJCC, 7th edition) for gastric cancer. The mean age of the patients at initial surgery was 63 years (range, 37-84 years); 62 men and 30 women were included in the present study. The mean duration of follow-up was 39 months (range, 1-73 months). The AJCC tumor stage distribution and vital status of the patients are shown in Table I. The study was approved by the Shanghai Jiao Tong University Medical School Institutional Review Board. Informed written consent to participate in the study was obtained from each of the patients before the entry into the study.

Immunohistochemistry staining. Immunohistochemistry (IHC) staining was performed using a highly sensitive streptavidin-biotin-peroxidase detection system with gastric cancer tissue microarrays. Rabbit anti-Rac1 at a dilution of
Table I. Demographic and clinicopathological parameters of enrolled patients.

Clinicopathological

characteristics

$\mathrm{N}$

$\%$

Age (years)

Median

Range

$37-84$

Gender

Male

62

67.4

Female

30

Local invasion

$\mathrm{T} 1 / 2$

25

27.2

T3

53

57.6

$\mathrm{T} 4$

14

15.2

Lymph node metastasis

Negative

25.0

Positive

Metastasis

M0

M1

10

10.9

TNM staging

I

11

II

30.4

III

46.7

IV

Lauren's type

Intestinal

Diffuse

Differentiation

Well, moderate

57

Poor, undifferentiated

35

38.0

1:50 (Abcam, Cambridge, MA, USA) was used as previously reported (25). Mayer's haematoxylin was used for counterstain. The slides were evaluated by a single board-certified pathologist (R.R.T.) who remained blinded to the clinical data using standard light microscopy.

Immunohistochemistry assessment. Expression status of Rac1 was determined by using a semi-quantitative scoring system based on the percentage of positive cells. The percentage of positive cells was divided into four grades (percentage scores): $<5 \%$ (0), $5-25 \%$ (1), $25-50 \%$ (2), $50-75 \%$ (3) and $>75 \%$ (4). All analyses were performed with the SPSS 17.0 software (SPSS Inc., Chicago, IL, USA). Correlation between Rac1 expression and clinical parameters was analyzed using the Pearson correlation coefficient analysis. Overall survival was calculated with the Kaplan-Meier method, and differences were compared by the log-rank test. P-value $<0.05$ was considered as statistically significant. 
Plasmids and transfection. The GFP-fused wild-type Rac1 (Rac1) and GFP-empty vector (vector) (26) were purchased from Addgene (Cambridge, MA, USA). For plasmid transfection, cells were seeded into 6-well plate and allowed to grow $24 \mathrm{~h}$ prior to transfection. Cells in each well were transfected with $4 \mu \mathrm{g}$ plasmid using Lipofectamine 2000 (Invitrogen). Clones stably transfected with either Rac1 or GFP vectors were selected through G418 culture.

Gastric cancer cell lines and cell culture. Gastric cancer cell lines AGS, NCI-N87, MKN45, MKN28, SGC-7901, KATO III, BGC823, MGC803, SNU-1 and SNU-16 were preserved in our institute. The cells were grown in RPMI-1640 medium containing $10 \%$ fetal bovine serum (FBS), penicillin and streptomycin (Gibco-BRL, Gaithersburgh, MD, USA). Cell proliferation was assessed by the 3-(4,5-dimethylthiazol-2-yl)2,5-diphenyltetrazolium (MTT) (Sigma, St. Louis, MO, USA) assay. Rac1 inhibitor NSC23766 was obtained from Merck Biosciences (Darmstadt, Germany). Final concentration of NSC23766 in treating cells was $100 \mu \mathrm{M}(27)$.

Soft agar and Matrigel 3D culture. Soft agar colony formation assay was performed by using $0.3 \%$ agar in complete medium with cells as the feeder layer and $0.6 \%$ agar in complete medium as the bottom layer. 3D Matrigel culture was performed using Matrigel matrix (BD Biosciences, San Jose, CA, USA).

Reverse transcription and quantitative real-time $P C R$ ( $q R T-P C R)$. Total RNA was isolated from cultured cells using the RNeasy mini kit (Qiagen) and cDNA was synthesized with oligo (dT) primers by using of a SuperScript First-strand cDNA Synthesis kit (Invitrogen) according to the manufacturer's protocols. Gene expression was assessed by qRT-PCR using an Applied Biosystems 7500 Fast Sequence detection system (Life Technologies, Carlsbad, CA, USA). The PCR reaction mixture consisted of QuantiTect SYBR-Green PCR Master Mix (2x QuantiTect SYBR-Green kit, contains HotStart Taq ${ }^{\circledR}$ DNA polymerase, QuantiTect SYGB-Green PCR buffer, dNTP mix, SYGB I, Rox passive reference dye and $5 \mathrm{mM} \mathrm{MgCl}_{2}$ ) (Qiagen), $0.5 \mu \mathrm{mol} / 1$ of each primer and cDNA. The transcript of the housekeeping gene, glyceraldehyde-3-phosphate dehydrogenase (GAPDH) gene was used as endogenous control to normalize expression data. The comparative $\mathrm{Ct}$ (threshold cycle) method was used to calculate the relative changes in gene expression. The primers used to amplify Rac1 were: 5'-ATGTCCGTGCAAAGTGGTATC-3' (forward) and 5'-CTCGGATCGCTTCGTCAAACA-3' (reverse). Primers to amplify GAPDH were 5'-AATGGG CAGCCGTTAGGAAA-3' (forward) and 5'-GCCCAATAC GACCAAATCAGAG-3' (reverse).

Immunofluorescence imaging. Immunofluorescence images were visualized using Olympus BX50 microscope (Olympus Opticol Co., Tokyo, Japan), images were taken using Nikon Digital Sight DS-U2 (Nikon, Tokyo, Japan), and NIS elements F3.0 software was used (Nikon). Confocal images were taken by inverted Zeiss LSM 710 confocal microscope (40x oil lens) (Carl Zeiss, Oberkochen, Germany). Zen 2009 Light Edition (Carl Zeiss) was used for the measurement of images.
Cell migration and invasion assays. Cell migration was analyzed by a Transwell chamber assay. Cell invasion assays were performed using BD BioCoat ${ }^{\mathrm{TM}}$ Matrigel $^{\mathrm{TM}}$ Invasion Chambers. FCS (10\%) was used as the chemoattractant. Cells on the lower surface of the insert were fixed and stained followed by counting under a light microscope. Cells were visualized using Olympus BX50 microscope (Olympus Opticol Co.), images were taken using Nikon Digital Sight DS-U2 (Nikon), and NIS elements F3.0 software was used (Nikon).

Cell lysis and western blot analysis. Cells were incubated for $4.5 \mathrm{~h}$ after transfection, washed once with PBS, and incubated an additional $1.5 \mathrm{~h}$ in DMEM lacking serum. Cells were washed twice with cold PBS and lysed with 150-200 $\mu$ l HEPES lysis buffer [50 mM HEPES (pH 7.0), $150 \mathrm{mM} \mathrm{NaCl,} 1 \%$ Triton $\mathrm{X}-100,10 \%$ glycerol, $50 \mathrm{mM} \mathrm{NaF}$ and $\left.1 \mathrm{mM} \mathrm{Na} \mathrm{VO}_{4}\right]$ supplemented with protease inhibitors $(5 \mu \mathrm{g} / \mathrm{ml}$ leupeptin, $5 \mu \mathrm{g} / \mathrm{ml}$ aprotinin, $1 \mu \mathrm{g} / \mathrm{ml}$ pepstatin and $100 \mu \mathrm{M}$ PMSF). Lysates were cleared at maximum speed in a microcentrifuge at $4^{\circ} \mathrm{C}$, and the protein concentration of each sample was determined using the Bio-Rad protein reagent. For western blots, proteins were resolved on $12 \%$ SDS-PAGE gels and transferred to PVDF membrane. Antibodies against Rac1 and GAPDH were purchased from Abcam.

Activity of Racl assay. The activation of Rac1 was measured using Rac1 Activation Assay Biochem kit (Cytoskeleton, Denver, CO, USA). Briefly, cell lysates were collected using lysis buffer from the kit. The activated forms of Rac1 were combined by PAK-PBD affinity beads. Beads were centrifuged and activate GTPases were pulled-down in the bead pallets. Bound GTPases were eluted by SDS buffer and analyzed by SDS-PAGE and western blotting. Racl levels were analyzed by the specific antibodies.

In vivo tumorigenesis and metastasis. Male BALB/c nu/nu nude mice (Institute of Zoology Chinese Academy of Sciences, Shanghai, China), were housed at a specific pathogen-free environment in the Animal Laboratory Unit, School of Medicine, Shanghai Jiao Tong University, China. Mice received humane care and the study protocols were approved by the Animal Care and Use Committee and conducted in accordance with the Guide for the Care and Use Laboratory Animals of Ruijin Hospital, Shanghai Jiaotong University School of Medicine. Cells $\left(1 \times 10^{6}\right)$ were subcutaneously injected into 4 -week-old male BALB/c mice. Tumor length (L) and width (W) were measured and tumor volume was calculated by the equation: Volume $=\left(\mathrm{W}^{2} \mathrm{x} \mathrm{L}\right) / 2$ (28). Mice were sacrificed 28 days after injection under anesthesia. Ten mice were used in each group. To produce peritoneal spreading experimental metastasis, $2 \times 10^{6}$ cells were injected into 5-week-old male BALB/c nude mice intraperitoneally. After 6 weeks, the mice were sacrificed under anesthesia. Ten mice were used in each group. Macrometastatics were visualized and counted.

Statistical analysis. The statistical analyses were performed using SPSS 13.0 software (SPSS Inc., Chicago, IL, USA). Values represent mean \pm standard deviation (SD) of samples measured in triplicate. Each experiment was repeated three 
A

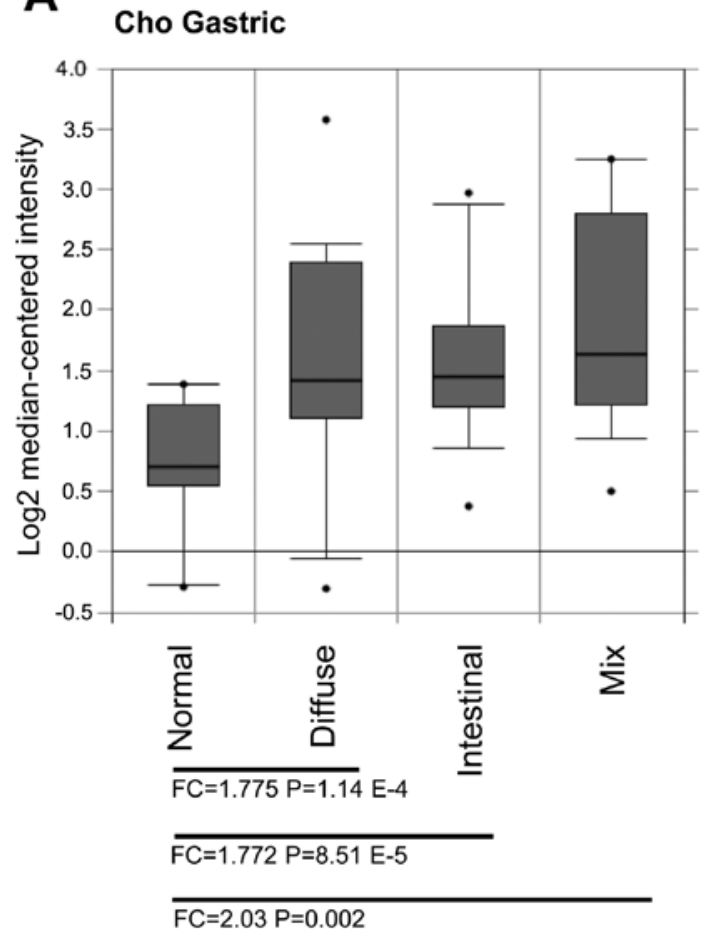

B

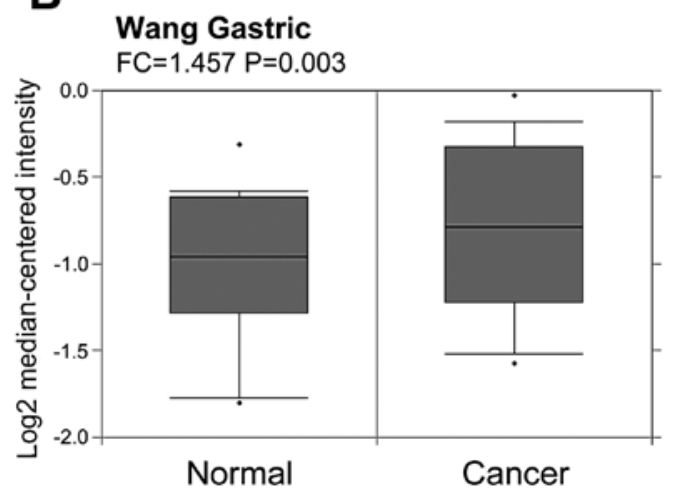

C Derrico dataset

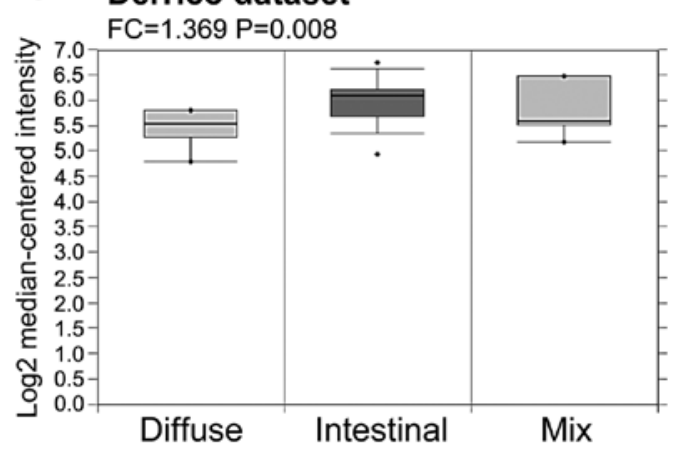

Ooi dataset
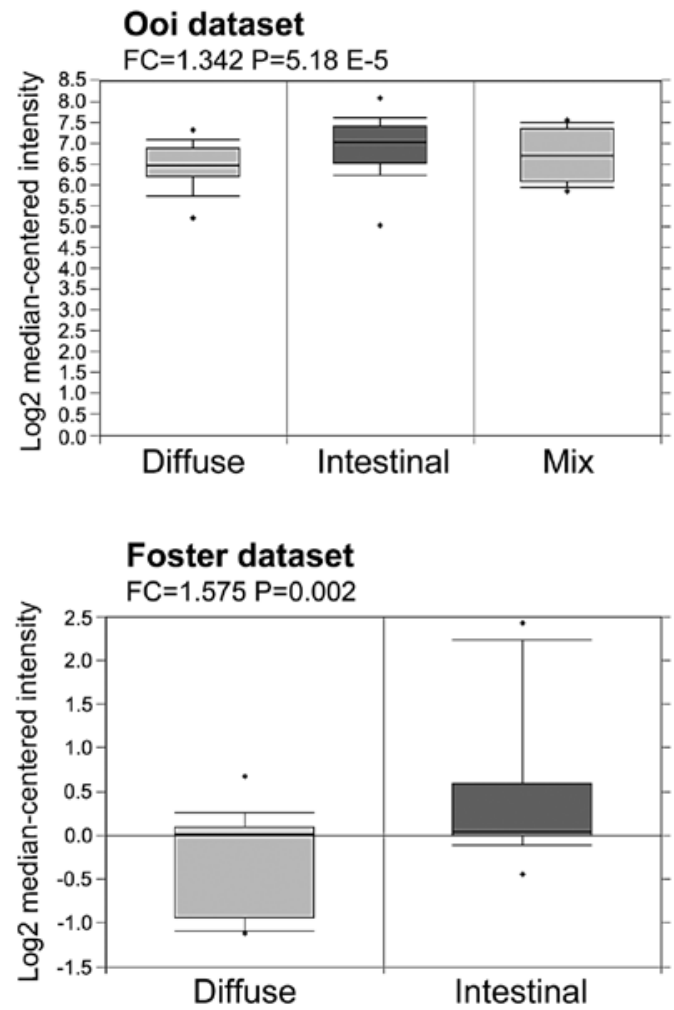

Figure 1. Expression of Rac1 mRNA levels in human gastric cancers using the Oncomine database. Analysis of Rac1 mRNA levels in human gastric cancer tissues compared with gastric normal tissues. The mRNA levels of Racl in human gastric cancers and normal gastric tissues in Cho et al (29) (A) and Wang et al (30) (B) datasets from the Oncomine database are shown. (C) Analysis of Rac1 mRNA levels in gastric cancer tissues with Lauren's classification. D'Errico et al (31), Ooi et al (32) and Foster et al (33) datasets from the Oncomine database are shown.

times, unless otherwise indicated. The significance of differences between experimental groups was analyzed using the Student's t-test and two-tailed distribution.

\section{Results}

Racl mRNA is overexpressed in human gastric cancer tissues. We first searched publicly available Oncomine database for the general information of Racl expression in gastric cancer tissues from previous publications. As we expected, Rac1 mRNA showed elevated levels compared with normal tissues. As shown in Fig. 1A and B, Rac1 mRNA levels were found to be higher in all the subtypes of gastric cancer compared with paired non-tumor tissues or normal gastric mucosal tissues in Cho et al (29) and Wang et al (30) datasets. Furthermore, by comparing Rac1 mRNA levels within different subtypes based on Lauren's classification, all the available datasets showed Racl mRNA levels were higher in intestinal-type than diffuse- and mix-types in D'Errico et al (31), Ooi et al (32), and Foster et al (33) datasets (Fig. 1C). These data suggest that Rac1 mRNA levels are elevated in gastric cancer tissues.

Racl protein is overexpressed in human gastric cancer tissues. We performed immunohistochemistry staining to examine the Rac1 protein expression in gastric cancer tissues. Gastric tumor and paired non-tumor tissues from 92 patients were stained. Rac1 positivity was observed in several crypt and isthmus epithelial cells (Fig. 2A) but not the surface epithelium (Fig. 2B) in normal gastric tissues. Positive Rac1 staining was $72.8 \%$ (67 of 92) in gastric cancer tissues, and $27.2 \%$ (25 of 92) of the samples showed negative staining. Rac1 positivity was 
A

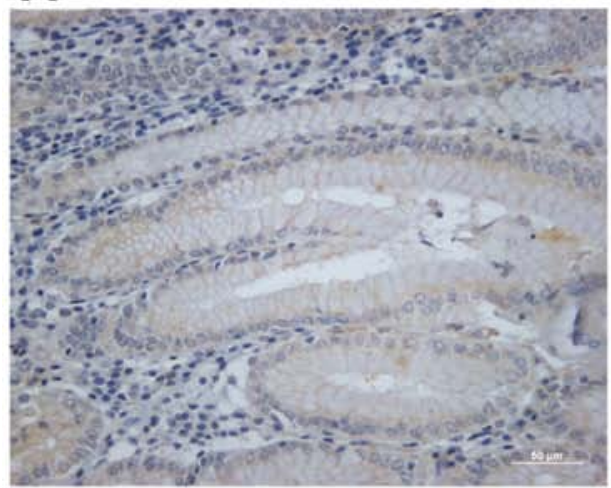

C

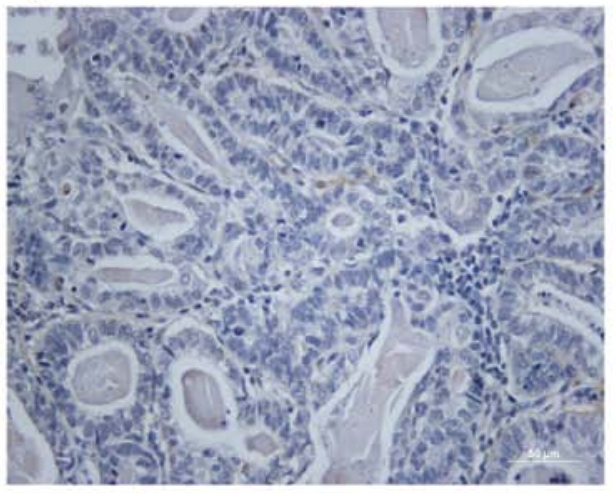

E

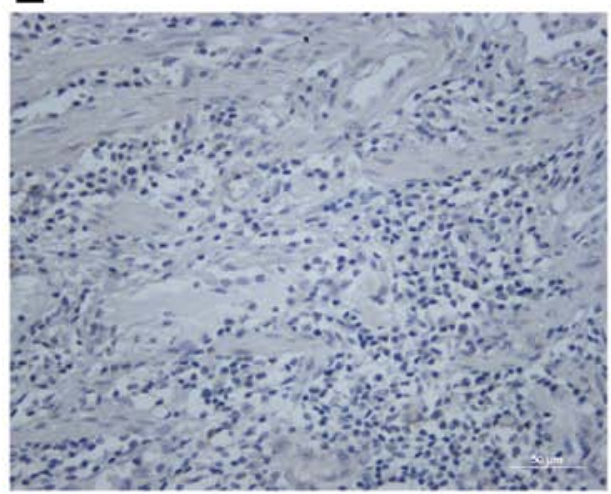

B

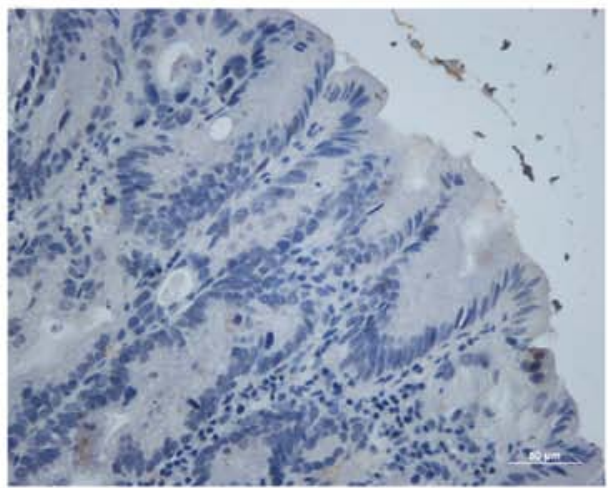

D

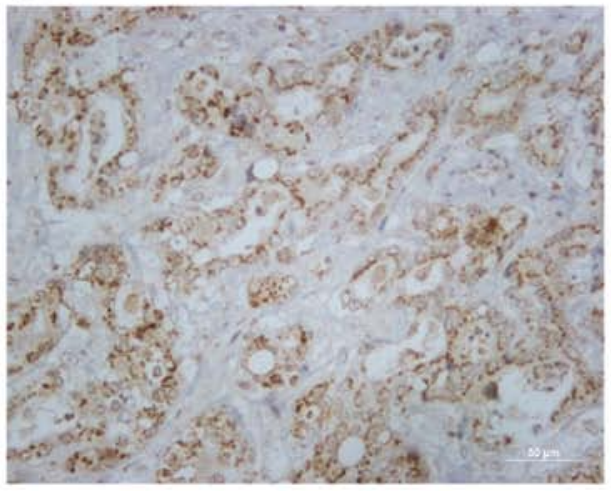

F

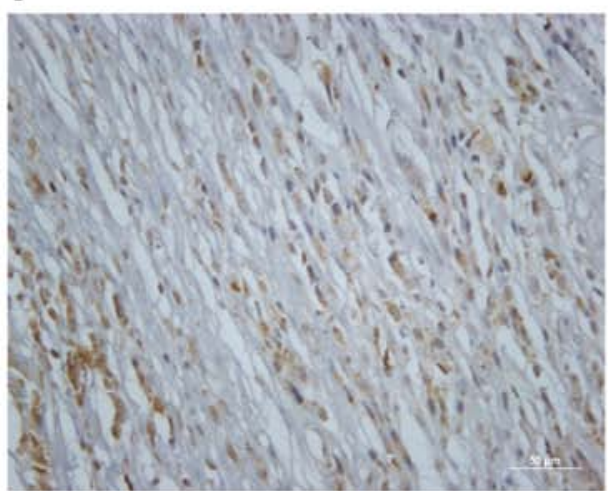

Figure 2. Expression of Rac1 protein levels in human gastric tumor and non-tumor tissues. Expression levels of Rac1 were examined by immunohistochemistry staining. Representative images showing non-tumor gastric tissues (A and B), intestinal-type gastric cancer (C and D), and diffuse-type gastric cancer (E and F) are shown. Rac1-negative (A, C and D) and positive (B, D and F) images are represented. Original magnification, $\mathrm{x} 200 . \mathrm{Bar}, 50 \mu \mathrm{m}$.

cytoplasmic, and in the membrane of the cancer cells (Fig. 2D and F) compared with negative staining (Fig. 2C and E). A significant correlation was found between positive expression of Racl with gender (female, $\mathrm{P}<0.001)$, local invasion (T3/4, $\mathrm{P}<0.001)$, lymph node metastasis $(\mathrm{P}<0.001)$, distant metastasis (M1, P<0.001), late TNM stage (III/IV, P=0.024), Lauren's classification (intestinal type, $\mathrm{P}<0.001$ ) and differentiation status (well and moderate, $\mathrm{P}<0.001$ ) (Table II). These data suggest that Rac1 is highly expressed in a subset of human gastric cancer which shows more malignant characteristics according to clinicopathological features.

Racl expression levels are correlated with shorter survival in gastric cancer patients. To investigate the prognostic significance of Rac1 in gastric cancer, we analyzed the corre- lation of Racl with patients' survival using Kaplan Meier analysis. The patients in the Rac1-positive group showed shorter overall survival than those in Racl-negative group (medium survival, 40.0 vs. 58.0 months, $\mathrm{P}=0.008$; Fig. 3). Our data suggested an association between Rac1 expression and a short overall survival in gastric cancer patients.

Expression of Racl in gastric cancer cell lines. We then analyzed the Racl expression in human gastric cancer cell lines and immortalized normal gastric epithelial cell line GES-1. We first performed qRT-PCR and immunoblotting to analyze the Rac1 mRNA and protein levels in gastric cancer cell lines and GES-1. In consistent with database analysis, we showed that Rac1 mRNA (Fig. 4A) and protein (Fig. 4B) levels were highly expressed in all gastric cancer cell lines compared to 
Table II. Protein expression and clinicopathological characteristics.

\begin{tabular}{l} 
Protein expression $(\mathrm{n})$ \\
\hline $\begin{array}{l}\text { Cases } \\
(\mathrm{n}=92)\end{array} \quad \begin{array}{c}\text { Negative } \\
(\mathrm{n}=25)\end{array} \quad \begin{array}{c}\text { Positive } \\
(\mathrm{n}=67) \quad \text { P-value }\end{array}$
\end{tabular}

\section{Age (years)}

$\begin{array}{lrrrr}60 & 38 & 6 & 32 & 0.092^{\mathrm{b}} \\ \geq 60 & 54 & 19 & 35 & \end{array}$

Gender

$\begin{array}{lrrrr}\text { Male } & 62 & 19 & 43 & <0.001^{\mathrm{b}} \\ \text { Female } & 30 & 6 & 24 & \\ \text { Local invasion } & & & & \\ \text { T1/2 } & 25 & 14 & 11 & <0.001^{\mathrm{a}} \\ \text { T3 } & 53 & 7 & 46 & \\ \text { T4 } & 14 & 4 & 10 & \end{array}$

Lymph node metastasis

\begin{tabular}{lrrrr} 
Negative & 23 & 15 & 8 & $<0.001^{\mathrm{b}}$ \\
Positive & 69 & 10 & 59 & \\
Metastasis & & & & \\
M0 & 82 & 24 & 58 & $<0.001^{\mathrm{b}}$ \\
M1 & 10 & 1 & 9 & \\
TNM staging & & & & \\
I/II & 39 & 15 & 24 & $0.024^{\mathrm{b}}$ \\
III/IV & 53 & 10 & 43 & \\
Lauren's type & & & & \\
Intestinal & 54 & 12 & 42 & $<0.001^{\mathrm{b}}$ \\
$\quad$ Diffuse & 38 & 13 & 25 & \\
$\quad$ Differentiation & & & & \\
$\quad$ Well, moderate & 57 & 14 & 43 & $<0.001^{\mathrm{b}}$ \\
$\quad$ Poor, undifferentiated & 35 & 11 & 24 & \\
\hline
\end{tabular}

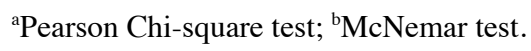

GES-1. These data demonstrate that Rac1 is highly expressed in gastric cancer cell lines.

Overexpression of Racl in gastric cancer cells increases cell proliferation in monolayer and $3 D$ culture. We next explored the role of Rac1 in gastric cancer cells. To do so, we generated Rac1 overexpression models using SGC-7901 (Fig. 5A) and BGC823 cell line (data not shown), which expressed moderate and low levels of Rac1, respectively. Analyzed by quantitative RT-PCR and western blotting, Rac1 mRNA (Fig. 5A, left panel) and protein (Fig. 5A, right panel) levels were increased in Racl stable transfectants compared with vector-control transfectants. To investigate the role of Rac1 in the growth of gastric cancer cells, we first performed a cell proliferation assay in monolayer culture. As shown in Fig. 5B, Rac1 overexpression significantly increased cell growth in monolayer culture. We then performed soft agar and Matrigel 3D culture. As expected, we found that Racl overexpression promoted the growth of SGC-7901 (Fig. 5C) and BGC823 (data not shown)

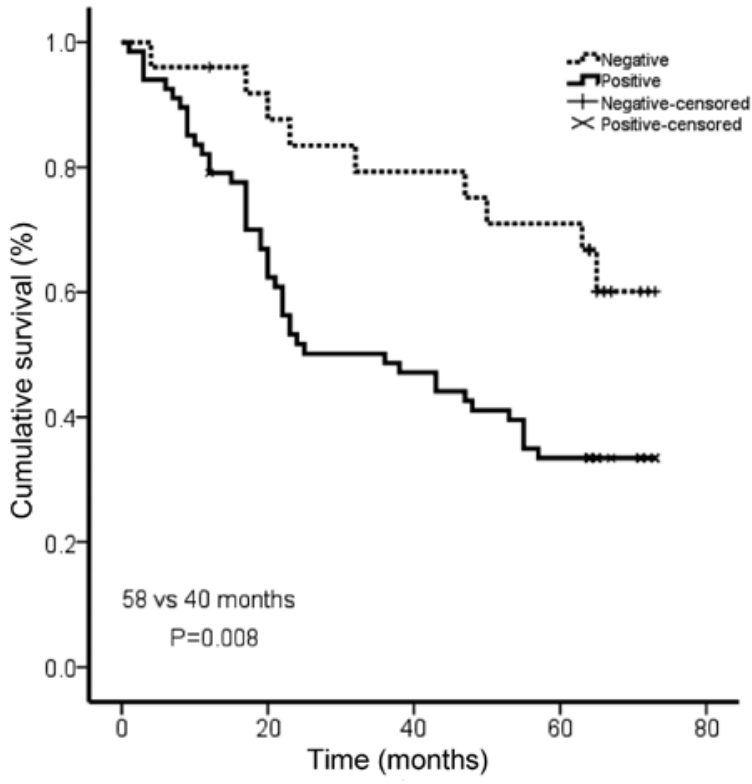

Figure 3. Survival analysis of Rac1 protein in gastric cancer specimens. Kaplan-Meier analysis and the log-rank test identified Rac1 as significant associated with cancer specific survival in gastric cancer patients.

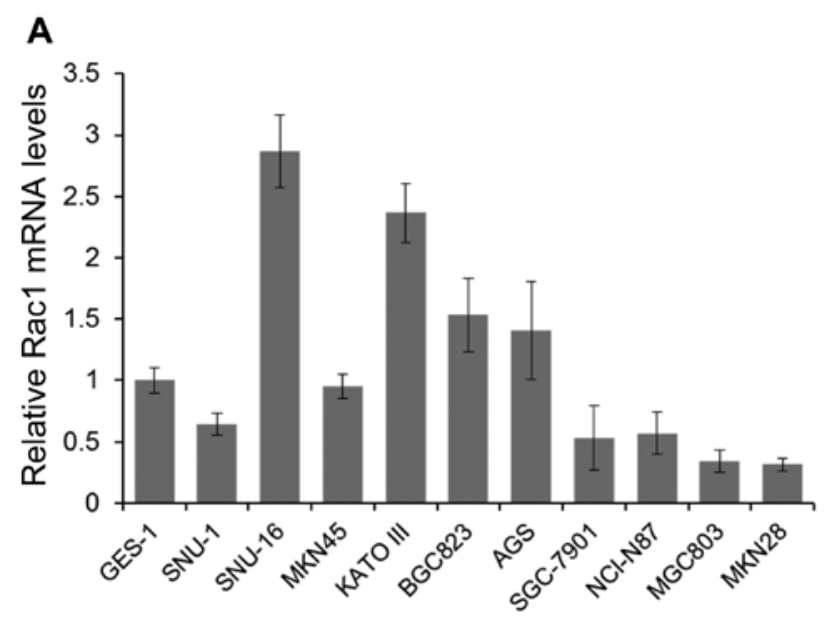

B

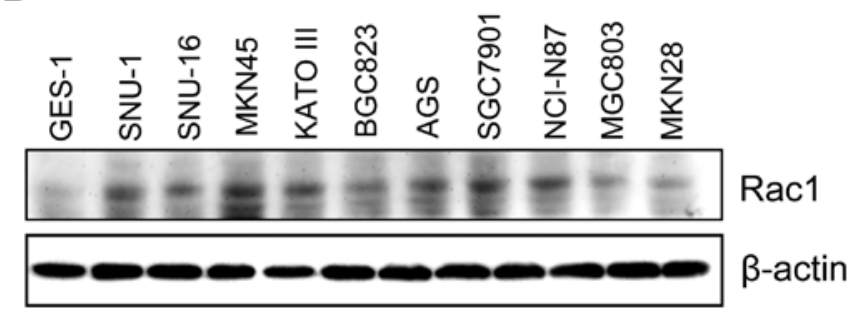

Figure 4. Rac1 was overexpressed in gastric cancer cell lines. Rac1 mRNA (A) and protein (B) levels in ten gastric cancer cell lines and immortalized normal gastric epithelial cell line GES-1. Data represent mean \pm SD of three independent experiments in the column plot.

gastric cancer cells in soft agar as suggested by larger colonies. Similarly, Racl overexpressing cells formed bigger spheres in Matrigel 3D culture (Fig. 5D). These data suggest that Rac1 overexpression increases cell proliferation in monolayer and $3 \mathrm{D}$ condition. 

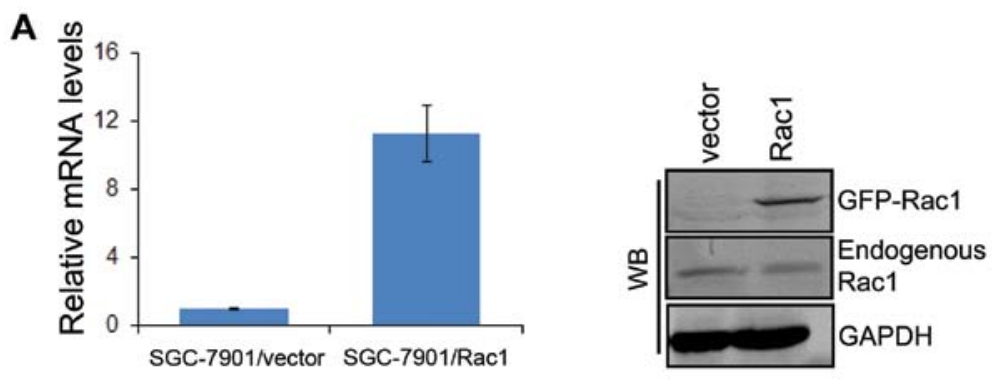

B

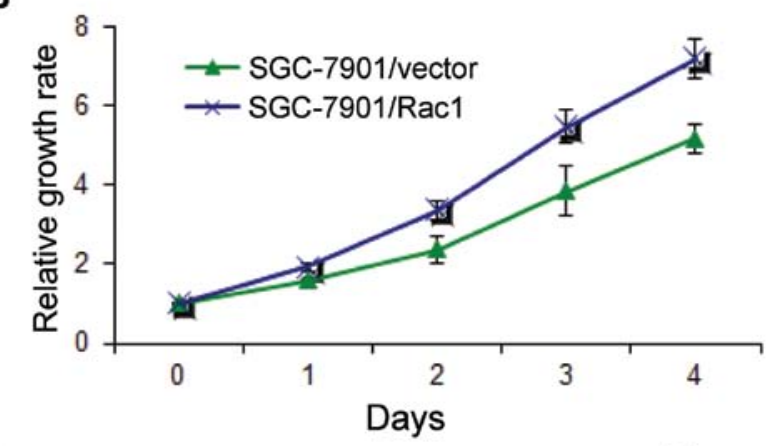

C

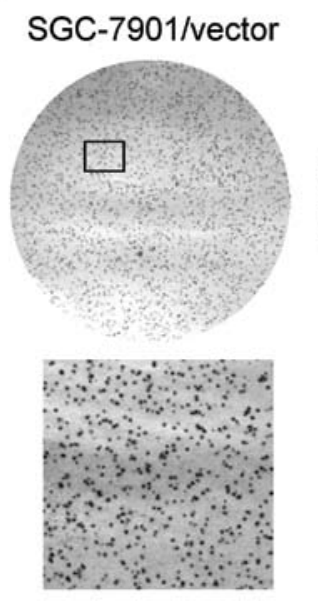

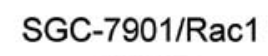
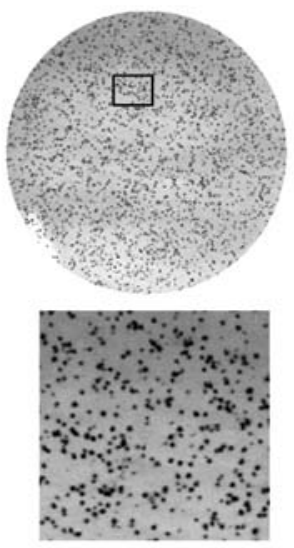

D

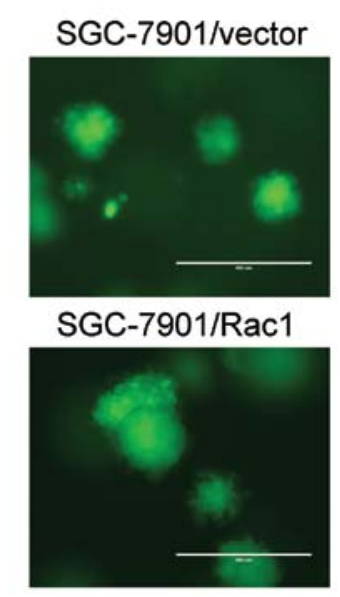

Figure 5. Rac1 overexpression promoted gastric cancer cell growth in vitro. (A) Racl mRNA (left panel) and protein (right panel) levels were increased in Rac1-overexpressed SGC-7901 cells. (B) Overexpression of Rac1 in SGC-7901 gastric cancer cells increased growth in monolayer culture. (C) Overexpression of Rac1 in SGC-7901 gastric cancer cells increased cell growth in soft agar. (D) Overexpression of Rac1 in SGC-7901 gastric cancer cells increased cell growth in 3D Matrigel.

Overexpression of Racl in gastric cancer cells increases cell migration and invasion. We observed a morphological alteration in Rac1-overexpressing cells, with abundant cell protrusions and membrane ruffling (Fig. 6A, arrows). This phenomenon suggested a highly aggressive feature. As Rac1 expression was positively correlated with the aggressiveness in gastric cancer specimens, we next asked whether overexpression of Racl affected the ability of gastric cancer migration and invasion. Boyden chamber assays were used to investigate the in vitro ability of migration and invasion in Rac1-overexpression and vector-control cells. As expected, Rac1-overexpression induced more cells to migrate through the Boyden chambers (Fig. 6B and C). Likewise, more cells in Rac1-overexpression group invaded through Matrigel-coated Boyden chambers than those in vector-control cells (Fig. 6B and $\mathrm{C}$ ). These data suggest that Racl promotes gastric cancer cell metastasis in vitro.
Inhibition of Racl activity abrogates the effects of Racloverexpression. Previous findings have suggested that the activity Racl is essential in mediating downstream effects on cell proliferation and migration/invasion. We first tested whether Rac1 activity was increased in SGC-7901 cells overexpressing Rac1. By using Racl activity assays, we found that GTP-bound-Rac1 (activated form of Rac1) was increased in Rac1-overexpressing cells compared with that in vectorcontrol cells (Fig. 7A, left panel). We next asked whether Rac1 activity was responsible for the increased proliferation and migration/invasion in gastric cancer cells. To this end, we used a chemical (NSC23766) that specifically inhibited Rac1 activity (34). When treated with NSC23766, the level of GTP-bound-Rac1 in SGC-7901/Rac1 cells dramatically dropped (Fig. 7A, right panel). Of note, Rac1 inhibitor NSC23766 induced an obvious morphological change in SGC-7901/Rac1 cells. As shown in Fig. 7B, NSC23766 
A SGC-7901/vector
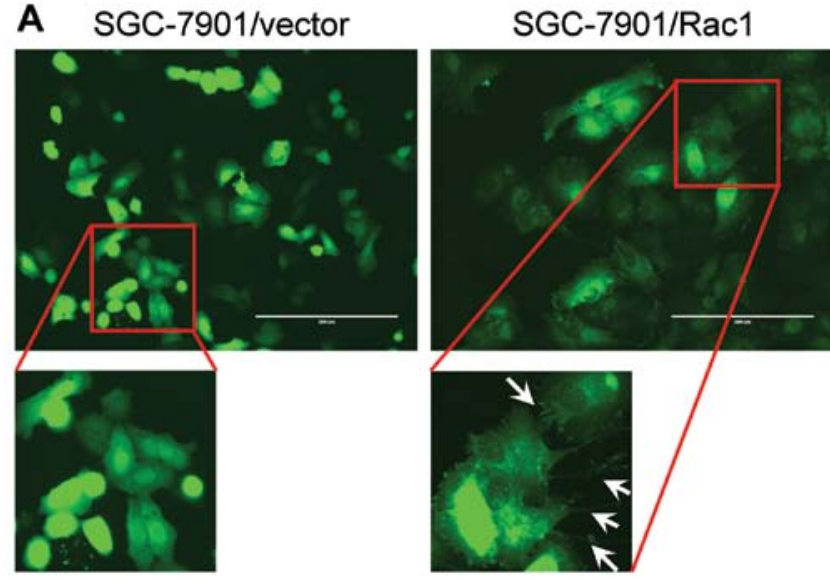

B
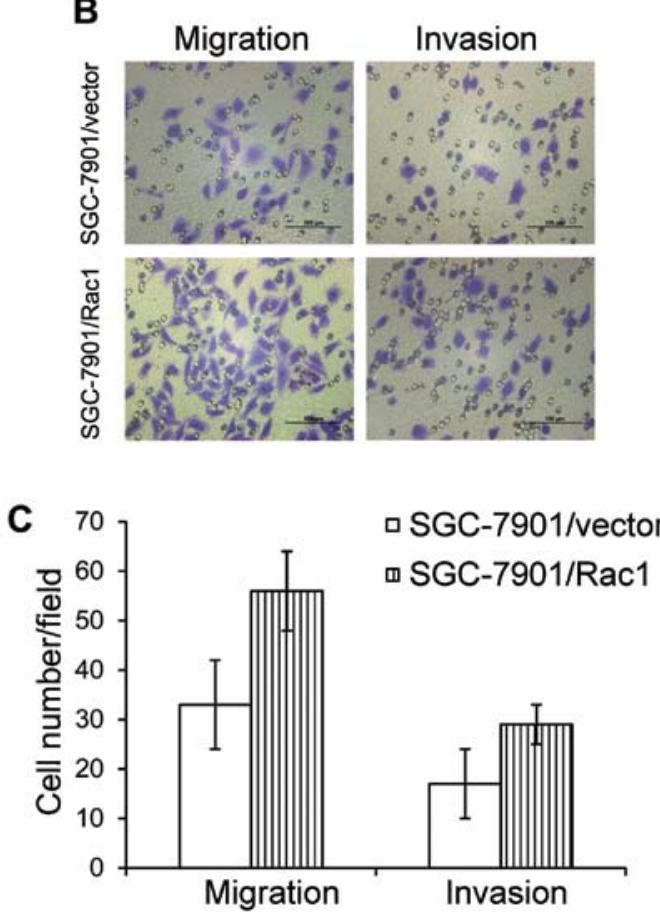

Figure 6. Rac1 overexpression promoted gastric cancer cell migration and invasion. (A) Racl overexpression rendered aggressive morphological alterations in SGC-7901 cells. Cells stably transfected with GFP-vector and GFP-Rac1 were observed under a fluorescence microscope. White arrows indicate cell membrane ruffling and protrusions. (B) Rac1 overexpression induced higher migration and invasion rate in SGC-7901 cells. Representative images of migrated/invaded cells are shown (original magnification, x200). The number of migrated/invaded cells is plotted (C).

treatment inhibited the formation of membrane ruffling and protrusions. We then asked whether Rac1 inhibition rendered cellular function alterations. As expected, NSC23766 treatment inhibited Rac1-overexpressing cell growth in 4-day observation period (Fig. 7C). Furthermore, Racl inhibition suppressed cell migrating and invading ability (Fig. 7D). These data suggest that the inhibitor of Racl activity abolishes Rac1-overexpression inducing cell morphological alterations and cellular functions.

Overexpression of Racl in gastric cancer cells increases in vivo tumorigenesis and metastasis. In order to examine the effects of Racl on the in vivo growth of gastric cancer cells,

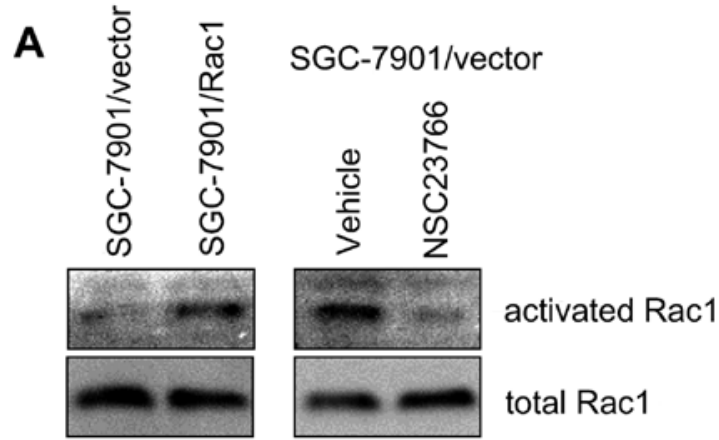

B
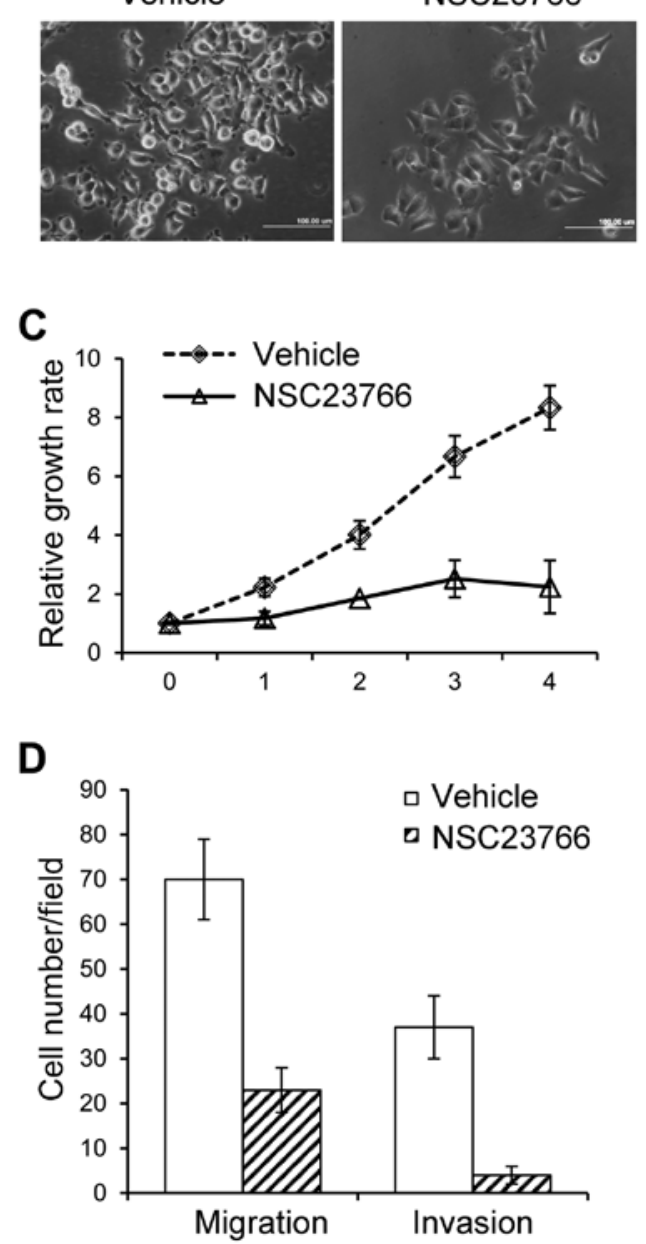

Figure 7. Inhibition of Rac1 activity abrogated the effect of Rac1 overexpression. (A) The Rac1 inhibitor NSC23766 blocked the activation of Rac1 in Rac1-overexpressed SGC-7901 cells. Activated Rac1 in SGC-7901/vector, SGC-7901/Rac1 as well as in vehicle or NSC23766 treated SGC-7901/Rac1 cells was performed by Racl activity assay (see Materials and methods) $24 \mathrm{~h}$ after treatment. Total Rac1 levels were used as loading control. (B) NSC23766 inhibited the aggressive phenotype. Representative images are shown of cells treated with vehicle or NSC23766 for $24 \mathrm{~h}$. Original magnification, x200. (C) NSC23766 inhibited SGC-7901/Rac1 growth. Relative growth rates (compared with day 0) are plotted. (D) NSC23766 inhibited the migrating and invading capacity of SGC-7901/Racl cells. The number of migrated/invaded cells $24 \mathrm{~h}$ after treatment is plotted.

we employed two experimental models. Control and Rac1overexpression SGC-7901 cells were injected subcutaneously into nude mice and tumor growth was examined. Mice injected with control and Rac1-overexpression cells formed similar 

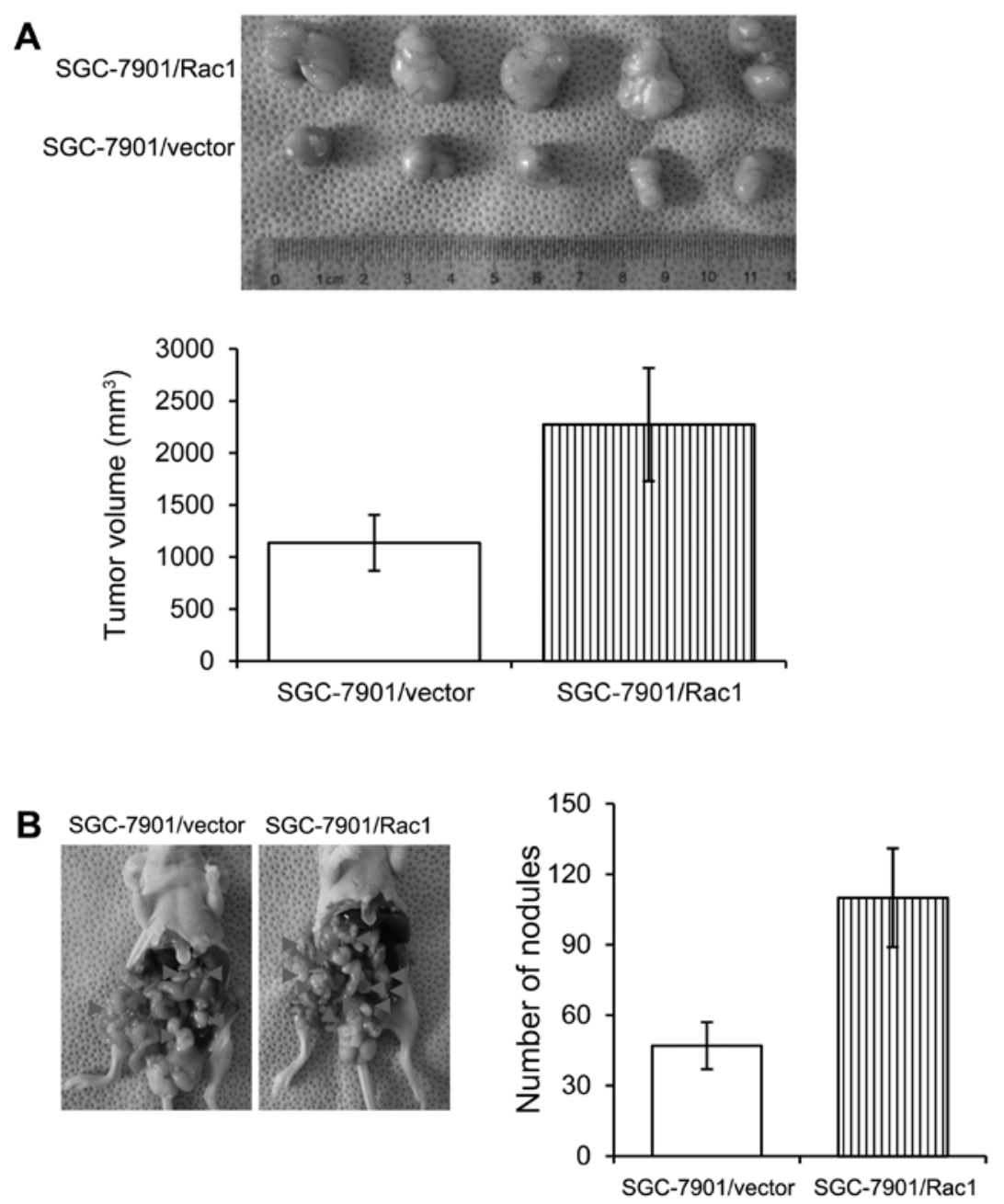

Figure 8. Rac1 overexpression enhanced tumorigenicity and metastasis of human gastric cancer cells using in vivo models. (A) Growth curves of tumors after injection of control and Rac1-overexpression SGC-7901 cells in nude mice. Data are shown as mean \pm SD $(n=10)$. Representative pictures show tumors at the day of scarifice. (B) Effects of Rac1 overexpression on suppressing peritoneal spreading and metastasis. Metastatic nodules are obvious in control group as indicted by the arrows. The number (mean \pm SD) of metastatic nodules in control and Rac1-overexpression group are plotted $(n=10)$.

size tumors within 37 days (Fig. 8A). As peritoneal spreading and metastasis are common in gastric cancer and are pivotal factors for its poor prognosis, we used a nude mouse model to investigate the influence of Racl levels on peritoneal metastasis. Consistent with in vitro observations, we found that Rac1-overexpression cells formed less metastatic nodules than control cells (Fig. 8B). Our data suggest that Racl overexpression increased in vivo tumorigenesis and metastasis.

\section{Discussion}

Rac1 is one of the Rho-family small guanine nucleotide triphosphate hydrolases. Racl regulates cellular functions such as cell proliferation, survival and invasiveness. In human cancers, Rac1 is considered as an oncogene which promotes malignant transformation and progression. Racl is the most studied Rac family protein in human cancers. Racl is a multifunctional protein that serves not only as a scaffolding protein but also as a special regulator of downstream effectors modulating a number of cellular responses. Rac1 is found to play a pivotal role in progression of cancer. In the present study, we evaluated the expression and clinical relevance of Rac1 in human gastric cancer specimens. We further explored the role of Rac1 in gastric cancer by using ectopic-overexpression models. In line with the clinical conclusions, we showed that Rac1 promoted cell proliferation and metastasis by in vitro and in vivo studies.

Metastasis has a major impact on the morbidity and mortality of patients with cancer (35). In the case of gastric cancer, vast majority of the patients are diagnosed at advanced stage. Identifying biomarkers that are functionally critical in metastasis is urgent in treating gastric cancer patients. Our studies discovered that high Rac1 levels were well correlated with gastric cancer malignancy and predicted poor survival in gastric cancer patients as assessed in clinical specimens. Our findings are consistent with previous studies which suggested a correlation of Rac1 with gastric cancer malignancy $(20,21)$. We also found that Racl was highly expressed in intestinal-type gastric cancer and positively correlated with tumor differentiation, local invasion and lymph node metastasis. Noteworthy, these pathological parameters reflect tumor malignancy and determine, at least partially, the outcome of patients. A robust line of studies have provided evidence supporting the crucial role of Rac1 in human cancers. Rac1 expression was associ- 
ated with invasion and metastasis in urothelial carcinoma (19). Rac1 promoted colon cancer progression through promotion of cell proliferation, survival and migration (36). Rac1 expression is correlated with tumorigenesis, aggressiveness and treatment resistance in a number of cancers such as lung $(37,38)$, breast cancer $(39,40)$ and melanoma $(23,41)$. These clinical investigations have shown the close correlation of Racl with human cancers.

Tumor cell invasion and migration are driven by continuous remodeling of the actin cytoskeleton which also provides cell shape maintaining cellular structure and polarization. The classic Racl activity is as a cytoskeleton organizer and is required for lamellipodia formation. Racl plays an important role in the pathobiology of various malignancy-related processes that promote tumor progression. Studies from diverse types of cancers have suggested the essential role of Rac1 in regulating tumor metastasis. Racl can be activated by multiple upstream signals and induce invadopodia formation and cause metastasis in breast cancer (15). Activated Racl promotes the assembly of cell surface integrin protein molecules in the surface of the head of the cells and passes regulative signals to the actin cytoskeleton, thus, inducing actin filaments to aggregate in the plasma membrane and form sheets of pseudopodia, leading to cell membrane multipolarization, which eventually affects the movement of cell migration. In our studies, Rac1-overexpressing gastric cancer cells exhibited obvious morphogenic changes showing more and longer cell lamellipodia protrusions, as well as enhanced cell migration and invasion. The current findings suggested that Rac1 activation occurred in the leading edge of the tumor. In agreement with the previous findings that Racl signaling is not required for the mode of amoeboid motility (42); we found that Rac1-overexpression caused more mesenchymal phenotype features such as extended protrusions in multiple directions. Our further findings that Rac1-overexpressing cells had elevated Racl activity, in line with previous findings, illustrated the role of Racl as a key regulator of cell mobility in gastric cancer.

Recently, a study on head and neck squamous cell carcinomas (HNSCC) showed that Racl is abundantly expressed in HNSCC tumor tissues and associated with lower early response rate as well as higher risk of tumor recurrences, while normal cells largely lack the Rac1 expression (43). They further found that inhibition of Racl activity could be useful in overcoming treatment resistance and could be proposed for HNSCC patients with primary or secondary chemoradioresistance (43). In addition, Kaneto et al (38) discovered that Rac1 inhibition was a potential therapeutic target for gefitinib-resistant non-small cell lung cancer (NSCLC). In agreement with the study of Kaneto et al, another group found that Rac1 suppression could suppress NSCLC stem cells and thus inhibit their tumorigenic activity (44). These investigations have provided insight into Racl signaling as therapeutic targets for human cancer treatment. In our experimental cell model, we found similar effects when Rac1-overexpressing cells were treated with NSC23766, a specific inhibitor of Rac1 activity. Rac1-overexpressing gastric cancer cell aggressiveness, as shown by proliferation, migration and invasion, was largely compromised by NSC23766. Rac1 appears to be a promising and relevant target for the develop- ment of novel anticancer drugs. Indeed, emerging evidence indicates that Rac1 regulates tumor cell motility, survival, and more importantly tumor angiogenesis (13). Because novel inhibitors targeting Rac1 activity have been developed by many groups (45) and Racl plays an essential role in gastric cancer cellular functions, Rac1 is a potential target for gastric cancer treatment.

In summary, our findings show the significant correlation of Rac1 in the patient outcome and the antitumor effect of Rac1 inhibition in gastric cancer cells. Our initial data support a central role of Rac1 in gastric tumorigenesis and progression. Rac1 shows potential as a biomarker for targeted therapy in gastric cancer treatment.

\section{Acknowledgements}

This study was supported by grants from the National Science Foundation of China (81372645), Shanghai Natural Science Foundation from municipal government (13ZR1425900), Shanghai Jiao Tong University School of Medicine Science and Technology Foundation (13XJ10035) and Fong Shu Fook Tong Foundation to J. Zhang. This study was also partially supported by the Chinese National High Tech Program (2012AA02A504, 2012AA02A203), the National Natural Science Foundation of China (81172329, 81372644) to Y. Yu.

\section{References}

1. Brenner H, Rothenbacher D and Arndt V: Epidemiology of stomach cancer. Methods Mol Biol 472: 467-477, 2009.

2. Ridley AJ: Rho GTPases and actin dynamics in membrane protrusions and vesicle trafficking. Trends Cell Biol 16: 522-529, 2006.

3. Karpel-Massler G, Westhoff MA, Zhou S, et al: Combined inhibition of HER1/EGFR and RAC1 results in a synergistic antiproliferative effect on established and primary cultured human glioblastoma cells. Mol Cancer Ther 12: 1783-1795, 2013.

4. Menard L, Parker PJ and Kermorgant S: Receptor tyrosine kinase c-Met controls the cytoskeleton from different endosomes via different pathways. Nat Commun 5: 3907, 2014.

5. Kumar R, Gururaj AE and Barnes CJ: p21-activated kinases in cancer. Nat Rev Cancer 6: 459-471, 2006.

6. Valente AJ, Yoshida T, Clark RA, Delafontaine P, Siebenlist U and Chandrasekar B: Advanced oxidation protein products induce cardiomyocyte death via Nox $2 / \mathrm{Rac} 1 /$ superoxidedependent TRAF3IP2/JNK signaling. Free Radic Biol Med 60: 125-135, 2013.

7. Shepelev MV, Chernoff J and Korobko IV: Rho family GTPase Chp/RhoV induces PC12 apoptotic cell death via JNK activation. Small GTPases 2: 17-26, 2011.

8. Foldynova-Trantirkova S, Sekyrova P, Tmejova K, et al: Breast cancer-specific mutations in CK1epsilon inhibit Wnt/betacatenin and activate the Wnt/Rac1/JNK and NFAT pathways to decrease cell adhesion and promote cell migration. Breast Cancer Res 12: R30, 2010.

9. Wang X, Zhang F, Chen F, et al: MEKK3 regulates IFN-gamma production in $\mathrm{T}$ cells through the Rac1/2-dependent MAPK cascades. J Immunol 186: 5791-5800, 2011.

10. Esufali S and Bapat B: Cross-talk between Rac1 GTPase and dysregulated Wnt signaling pathway leads to cellular redistribution of beta-catenin and TCF/LEF-mediated transcriptional activation. Oncogene 23: 8260-8271, 2004.

11. Goc A, Abdalla M, Al-Azayzih A and Somanath PR: Rac1 activation driven by 14-3-3zeta dimerization promotes prostate cancer cell-matrix interactions, motility and transendothelial migration. PLoS One 7: e40594, 2012.

12. Vader P, van der Meel R, Symons MH, et al: Examining the role of Racl in tumor angiogenesis and growth: a clinically relevant RNAi-mediated approach. Angiogenesis 14: 457-466, 2011. 
13. Ma J, Xue Y, Liu W, et al: Role of activated rac1/cdc42 in mediating endothelial cell proliferation and tumor angiogenesis in breast cancer. PLoS One 8: e66275, 2013.

14. Lin KT, Gong J, Li CF, et al: Vav3-rac1 signaling regulates prostate cancer metastasis with elevated Vav3 expression correlating with prostate cancer progression and posttreatment recurrence. Cancer Res 72: 3000-3009, 2012.

15. Lin CW, Sun MS, Liao MY, et al: Podocalyxin-like 1 promotes invadopodia formation and metastasis through activation of Rac1/ Cdc42/cortactin signaling in breast cancer cells. Carcinogenesis 35: 2425-2435, 2014.

16. Schnelzer A, Prechtel D, Knaus U, et al: Rac1 in human breast cancer: overexpression, mutation analysis, and characterization of a new isoform, Raclb. Oncogene 19: 3013-3020, 2000.

17. Fritz G, Just I and Kaina B: Rho GTPases are over-expressed in human tumors. Int J Cancer 81: 682-687, 1999.

18. Zhu G, Wang Y, Huang B, et al: A Rac1/PAK1 cascade controls beta-catenin activation in colon cancer cells. Oncogene 31: 1001-1012, 2012

19. Kamai T, Shirataki H, Nakanishi K, et al: Increased Rac1 activity and Pak1 overexpression are associated with lymphovascular invasion and lymph node metastasis of upper urinary tract cancer. BMC Cancer 10: 164, 2010.

20. Zhan H, Liang H, Liu X, Deng J, Wang B and Hao X: Expression of Rac1, HIF-1alpha, and VEGF in gastric carcinoma: correlation with angiogenesis and prognosis. Onkologie 36: 102-107, 2013.

21. Wu YJ, Tang Y, Li ZF, et al: Expression and significance of Rac1, Pak1 and Rock1 in gastric carcinoma. Asia Pac J Clin Oncol 10: e33-e39, 2013

22. Kato T, Kawai K, Egami Y, Kakehi Y and Araki N: Rac1dependent lamellipodial motility in prostate cancer PC-3 cells revealed by optogenetic control of Rac1 activity. PLoS One 9: e97749, 2014

23. Bauer NN, Chen YW, Samant RS, Shevde LA and Fodstad O Rac1 activity regulates proliferation of aggressive metastatic melanoma. Exp Cell Res 313: 3832-3839, 2007.

24. Jun Cho H, Kim IK, Park SM, et al: VEGF-C mediates RhoGDI2 induced gastric cancer cell metastasis and cisplatin resistance. Int J Cancer 135: 1553-1563, 2014.

25. Zeng W, Fu K, Quintanilla-Fend L, Lim M, Ondrejka S and Hsi ED: Cyclin D1-negative blastoid mantle cell lymphoma identified by SOX11 expression. Am J Surg Pathol 36: 214-219, 2012

26. Subauste MC, Von Herrath M, Benard V, et al: Rho family proteins modulate rapid apoptosis induced by cytotoxic T lymphocytes and Fas. J Biol Chem 275: 9725-9733, 2000.

27. Wang X, Deng Y, Mao Z, et al: CCN1 promotes tumorigenicity through Rac1/Akt/NF-kappaB signaling pathway in pancreatic cancer. Tumour Biol 33: 1745-1758, 2012.

28. Safina A, Vandette E and Bakin AV: ALK5 promotes tumor angiogenesis by upregulating matrix metalloproteinase-9 in tumor cells. Oncogene 26: 2407-2422, 2007.

29. Cho JY, Lim JY, Cheong JH, et al: Gene expression signaturebased prognostic risk score in gastric cancer. Clin Cancer Res 17: 1850-1857, 2011

30. Wang Q, Wen YG, Li DP, et al: Upregulated INHBA expression is associated with poor survival in gastric cancer. Med Oncol 29: 77-83, 2012.
31. D'Errico M, de Rinaldis E, Blasi MF, et al: Genome-wide expression profile of sporadic gastric cancers with microsatellite instability. Eur J Cancer 45: 461-469, 2009.

32. Ooi $\mathrm{CH}$, Ivanova $\mathrm{T}, \mathrm{Wu} \mathrm{J}$, et al: Oncogenic pathway combinations predict clinical prognosis in gastric cancer. PLoS Genet 5 : e1000676, 2009.

33. Forster S, Gretschel S, Jons T, Yashiro M and Kemmner W: THBS4, a novel stromal molecule of diffuse-type gastric adenocarcinomas, identified by transcriptome-wide expression profiling. Mod Pathol 24: 1390-1403, 2011.

34. Gao Y, Dickerson JB, Guo F, Zheng J and Zheng Y: Rational design and characterization of a Rac GTPase-specific small molecule inhibitor. Proc Natl Acad Sci USA 101: 7618-7623, 2004.

35. Bid HK, Roberts RD, Manchanda PK and Houghton PJ: RAC1: an emerging therapeutic option for targeting cancer angiogenesis and metastasis. Mol Cancer Ther 12: 1925-1934, 2013.

36. Leve F and Morgado-Diaz JA: Rho GTPase signaling in the development of colorectal cancer. J Cell Biochem 113: 2549-2559, 2012.

37. Oleinik NV, Helke KL, Kistner-Griffin E, Krupenko NI and Krupenko SA: Rho GTPases RhoA and Rac1 mediate effects of dietary folate on metastatic potential of A549 cancer cells through the control of cofilin phosphorylation. J Biol Chem 289: 26383-26394, 2014.

38. Kaneto N, Yokoyama S, Hayakawa Y, Kato S, Sakurai H and Saiki I: RAC1 inhibition as a therapeutic target for gefitinibresistant non-small-cell lung cancer. Cancer Sci 105: 788-794, 2014.

39. Elsayed HE, Akl MR, Ebrahim HY, et al: Discovery, optimization, and pharmacophore modeling of oleanolic acid and analogues as breast cancer cell migration and invasion inhibitors through targeting Brk/Paxillin/Racl Axis. Chem Biol Drug Des: Jun 20,2014. doi: 10.1111/cbdd.12380 (Epub ahead of print)

40. BaugherPJ,Krishnamoorthy L,Price JEand Dharmawardhane SF: Rac1 and Rac3 isoform activation is involved in the invasive and metastatic phenotype of human breast cancer cells. Breast Cancer Res 7: R965-R974, 2005.

41. Watson IR, Li L, Cabeceiras PK, et al: The RAC1 P29S hotspot mutation in melanoma confers resistance to pharmacological inhibition of RAF. Cancer Res 74: 4845-4852, 2014.

42. Fackler OT and Grosse R: Cell motility through plasma membrane blebbing. J Cell Biol 181: 879-884, 2008.

43. Skvortsov S, Dudas J, Eichberger P, et al: Racl as a potential therapeutic target for chemo-radioresistant head and neck squamous cell carcinomas (HNSCC). Br J Cancer 110: 2677-2687, 2014.

44. Akunuru S, Palumbo J, Zhai QJ and Zheng Y: Racl targeting suppresses human non-small cell lung adenocarcinoma cancer stem cell activity. PLoS One 6: e16951, 2011.

45. Cardama GA, Comin MJ, Hornos L, et al: Preclinical development of novel Rac1-GEF signaling inhibitors using a rational design approach in highly aggressive breast cancer cell lines. Anticancer Agents Med Chem 14: 840-851, 2014. 